\title{
Devising the guidelines: the concept of uniportal video-assisted thoracic surgery-instrumentation and operatory room staff
}

\author{
Mahmoud Ismail ${ }^{1}$, Dania Nachira ${ }^{2}$ \\ ${ }^{1}$ Department of Thoracic Surgery, Klinikum Ernst von Bergmann Potsdam, Academic Hospital of the Charité-Universitätsmedizin Humboldt \\ University Berlin, Potsdam, Germany; ${ }^{2}$ Department of Thoracic Surgery, Fondazione Policlinico Universitario "A. Gemelli”, IRCCS, Università \\ Cattolica del Sacro Cuore, Rome, Italy \\ Contributions: (I) Conception and design: None; (II) Administrative support: None; (III) Provision of study materials or patients: None; (IV) \\ Collection and assembly of data: None; (V) Data analysis and interpretation: None; (VI) Manuscript writing: All authors; (VII) Final approval of \\ manuscript: All authors. \\ Correspondence to: Mahmoud Ismail, MD. Department of Thoracic Surgery, Klinikum Ernst von Bergmann Potsdam, Academic Hospital of the \\ Charité-Universitätsmedizin Humboldt University-Berlin, Potsdam, Germany. Email: mahmoud.ismail@klinikumevb.de.
}

\begin{abstract}
In the last years, uniportal video-assisted thoracic surgery (VATS) has been gaining more and more popularity, becoming a common procedure in several thoracic centers all over the world not only for minor procedures but also for major and complex cases. This technique combines the advantages of the less invasiveness with the oncological principles of open surgery. A standardization of the different peri- and intraoperative steps can help in the establishment and development of this technique. The aim of this paper is to set the basic steps for operating room set-up and instrumentation for starting or improving a uniportal VATS program in thoracic surgery centers.
\end{abstract}

Keywords: Uniportal video-assisted thoracic surgery (uniportal VATS); instrumentations; operating room set-up; uniportal VATS program; tips and tricks

Submitted Apr 08, 2019. Accepted for publication Jun 27, 2019.

doi: $10.21037 /$ jtd.2019.08.69

View this article at: http://dx.doi.org/10.21037/jtd.2019.08.69

\section{Introduction}

Since the first publication in 1924 by Singer and Graham on minimally invasive thoracic surgery, the main concerns about minimally invasive techniques were the necessity of new set-up and instrumentation to face up with technical difficulties (1).

During the last decades, several innovations were introduced in all surgical fields, allowing the establishment of video-assisted thoracic surgery (VATS), as it is conceived today, and the first VATS lobectomies in the 1990s (2-4).

And nowadays international and national guidelines (5-7) suggest VATS as the technique of choice for the treatment of early stage lung cancer, for the faster postoperative recovery of the patients due to less pain and physical stress.

On the wave of less and less invasiveness, the number and the length of incision have been reduced during the years in
VATS surgery, going from "Triportal" VATS to "Biportal" VATS till the one-incision technique called "Uniportal" VATS. The first time this technique was described for minor procedures and lung resections was in $1998(4,8,9)$. Since that, several series and studies were published on minor lung resections. But the first major lung resection performed in uniportal VATS was published by GonzalesRivas in 2010 (10).

As in any new and revolutionary technique, the standardization of the procedure in perioperative and intraoperative aspects is the crucial point not only for the best development of the technique itself but also for its easy spreading among surgeons' community.

\section{Perioperative management}

Basically, the preoperative pathway for a uniportal VATS 


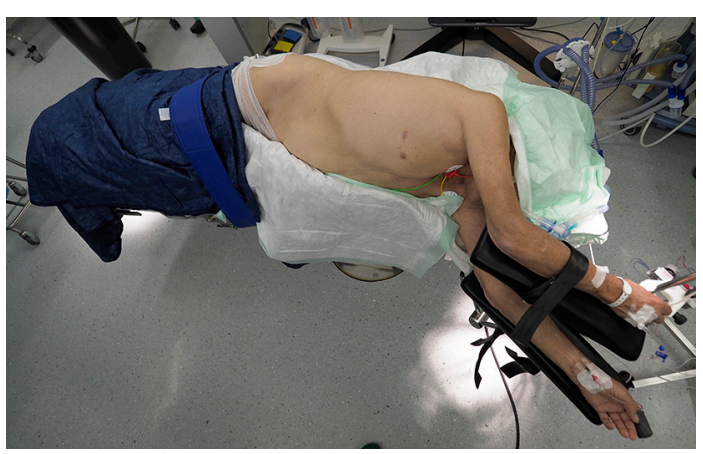

Figure 1 Uniportal VATS patient's position on surgical table. VATS, video-assisted thoracic surgery.

procedure is the same of any other thoracic procedure. Nowadays, the preferred pathway in surgery is the application of the fast-track concept and ERAS protocols $(11,12)$, optimizing the recovery of the patient and allowing an early and safe return to daily life. Therefore, the combination of both concepts, which share the same aim of fast and safe recovery, can optimize the outcome of patients.

Uniportal VATS is usually performed under general anesthesia and single-lung ventilation, however in some specialized centers it can be even performed in nonintubated fashion (13).

In general, there is no need for central venous catheters or peridural/epidural analgesia control systems to be placed preoperatively.

\section{Intraoperative settings and technical aspects}

\section{Patient position}

The patient is placed in a comfortable lateral decubitus position, with both arms flexed and stretched towards his head in order to provide also enough space to surgeons that stand on his ventral side (Figure 1).

The intercostal spaces can be better widened by flexing the surgical table in a wedge-shape position or putting a rolled blanket under patient's thorax at nipple level. For a better stabilization of the patient a vacuum mattress can be used. Thermal blankets are recommended for an optimal control of temperature during the anesthesia and a consequent early extubation of the patient after surgery.

New models of electric surgical tables also facilitate the position of patient by giving the possibility of tilting the patient some degrees on his ventral side, towards the surgeons, or in anti- Trendelenburg position. These movements of the table may also facilitate the exposure of some lung structures and prevent fatigue of the assistant in holding the camera.

\section{Operative-room staff positioning}

Usually both surgeons-the operator and the assistantoperate on the same side, standing on the ventral side of the patient and watching the same monitor opposite to them (Figure 2).

Some tips and tricks are useful in surgeons' position for ensuring more room and comfort to both of them during surgery.

When the surgeon is working on the cranial and middle part of the chest cavity, the assistant must stand on the caudal region of the patient. Conversely, if the surgeon is operating on the caudal part of chest cavity, standing on cranial side of patient is preferable for the assistant.

The scrub nurse must be always opposite surgeons looking at her own monitor, if available in the operating theater, for better assisting during surgery. Indeed, two monitors for the surgical staff would be the optimum for uniportal VATS, however in case of only one monitor available, it can be placed cranially to the patient.

All the cables (light cable, electric ones, suction tube...) must come from patient's back (nurse's side), fixed on his hip/shoulder depending on operating room setting, using the patient's hip as fulcrum for the movement of any cable (Figure 3). A methodical set-up like that one suggested let surgical staff operate using several instruments simultaneously without having tangle of wires on the operating field.

\section{Uniportal VATS incision}

A 2-4 cm single incision is usually done in the $\mathrm{V}$ intercostal space between the anterior and middle axillary line, but sometimes variations are necessary according to the position of the lesion and patient's anthropometric features (14). For lesions in upper lobes, central tumors or sleeve resections an incision in the IV intercostal space may provide better exposure and management.

The incision is performed according to muscle-sparing principles (the fibers of serratus anterior muscle are opened without being cut) and no rib spreading is necessary (14).

It is not necessary to enlarge the intercostal incision more than the skin one; this can prevent postoperative skin emphysema. 


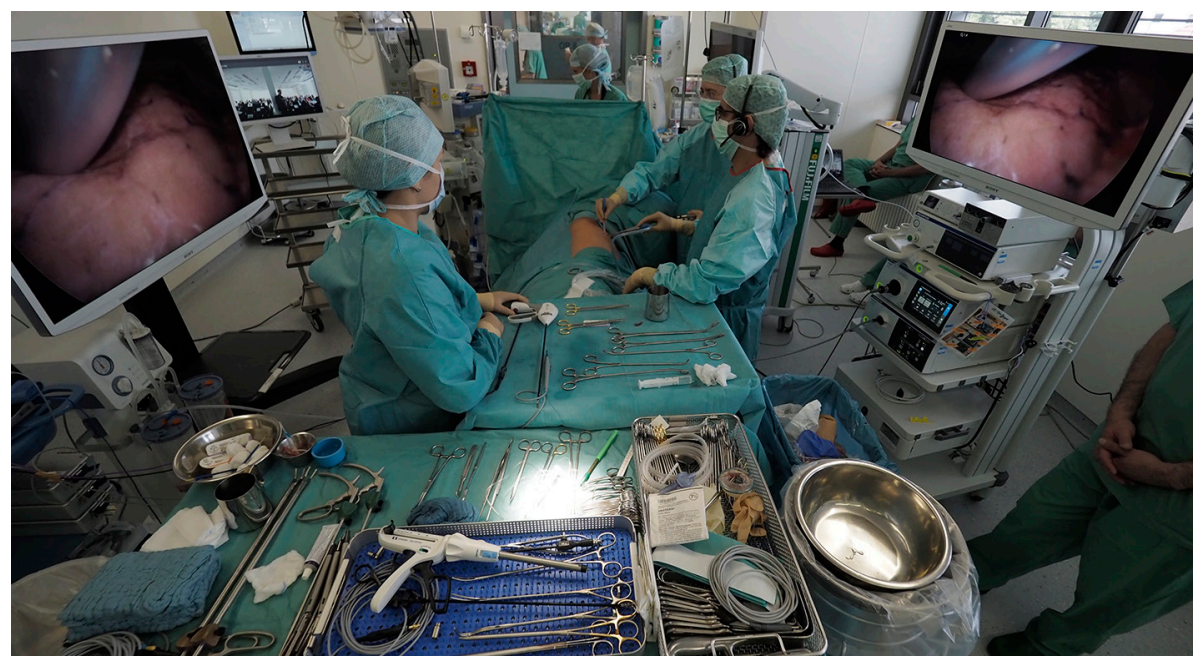

Figure 2 Staff position in operating room.

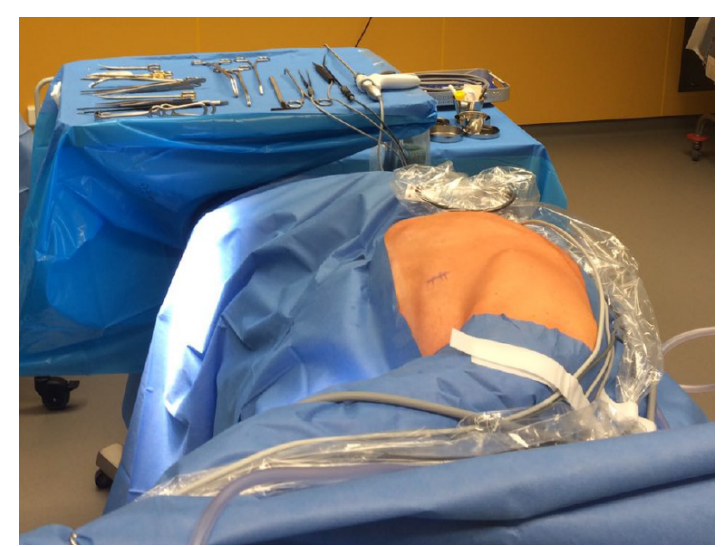

Figure 3 Operating field equipped for a uniportal VATS procedure. VATS, video-assisted thoracic surgery.

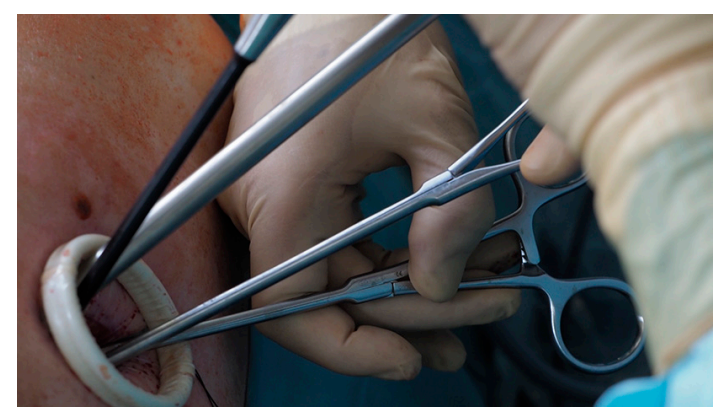

Figure 4 Aspect of uniportal VATS incision with wound protector placed and all working instruments inside. VATS, video-assisted thoracic surgery.

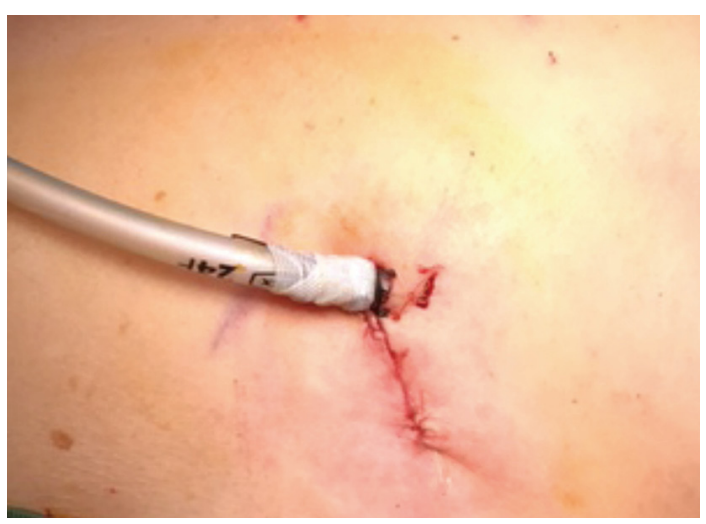

Figure 5 Placement of the drainage through the incision.

The use of a wound protector can favor the simultaneous introduction of different instruments, providing more space and preventing the soiling of the camera as well as decreasing the risk of wound infection (Figure 4). For better placement and sliding of the instrument through the wound protector a lubricating gel can be used.

No epidural/peridural catheter is necessary. At the end of the operation, a less invasive intercostal nerve block (14) or a serratus plane block (15) can be performed for a good postoperative pain control.

Only one chest tube is sufficient in the major part of the cases and it is inserted through the same incision and fixed in the superior part of it (the same place of the camera) (Figure 5). In particular cases, like pleural toilette for 

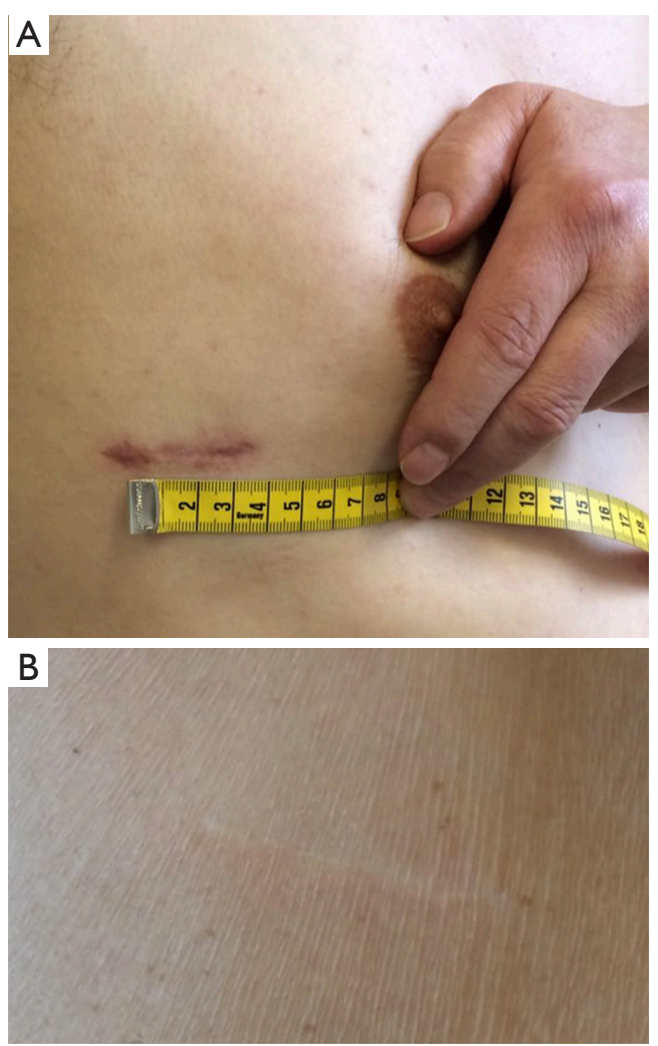

Figure 6 Aspect a uniportal VATS scar 1 week after the operation (A) and 9 months later (B). VATS, video-assisted thoracic surgery.

empyema, severe air leakage or bleeding, 2 chest tubes can be inserted through the same incision.

No intercostal stiches are necessary for closing the wound. It is sufficient to move the serratus muscle fibers with the fascia back together, fixing them with a running suture. For better cosmetic results, skin is closed by resorbable intradermal suture (Figure 6).

\section{Instrumentation}

For uniportal VATS procedures special instruments are necessary. They are curved instruments properly designed with a dual pivot point (proximal and distal) and a thin shaft. This particular shape allows the simultaneous introduction of several instrument through the small incision, without problems of fencing (13). Nowadays, some companies produce a wide range of uniportal instruments for any purpose (dissectors, ring forceps, node graspers, $45^{\circ}$ clip appliers etc.) and type of operation.

A $30^{\circ} 10$ or $5 \mathrm{~mm}$ camera is used for reaching any side of

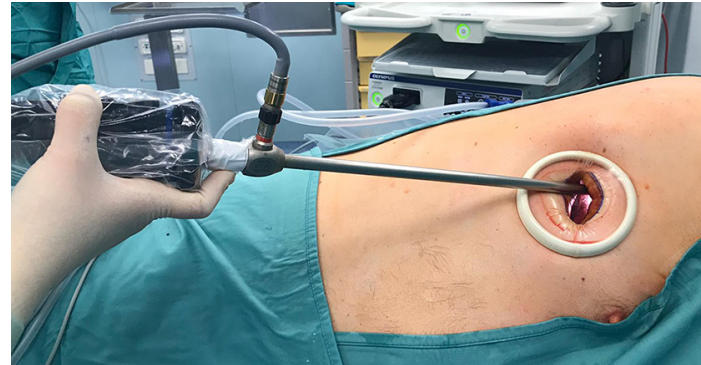

Figure 7 Position of the camera inside the incision.

the thorax or visualize any vascular or bronchial bifurcation through the rotation of the $30^{\circ}$ angle. A $10 \mathrm{~mm}$ camera can be suggested in some cases for having a clearer and brighter vision. In general, the camera must be always held in the upper part of the incision, while all other instruments must be introduced under it.

\section{Intraoperative tips and tricks}

The tilt of the camera shank does not exceed $30^{\circ}-40^{\circ}$ on the horizontal plane in order to prevent an excessive fatigue of assistant's shoulder in holding the camera (Figure 7). This can be achieved by tilting the operative table towards the surgeons and eventually some degrees in anti-Trendelenburg position.

Depending on his position with respect to the first operator, the assistant should be able to use the right or left hand to hold the camera, giving enough room to the first operator to work and helping in holding or moving instruments when necessary.

In general, the main working instrument in that step of the operation must be always introduced in the lower part of the incision, under any other instrument inserted before, for avoiding any problem of fencing (16).

Following this main rule, the ring forceps or graspers usually are held in the middle part of the incision, just under the camera and over the main working instrument used by the operator for that step of the operation (like a dissector or a stapler) (Figure 8).

The correct exposure of the lung and main structures to be dissected is the crucial point in each step of a uniportal VATS lobectomy. For example, in order to expose the hilum during a uniportal VATS upper lobectomy, a ring forceps must be placed on segment 3 parenchyma to retract it caudally and towards the back of the patient. On the other hand, to expose the lower vein, the lung parenchyma must 


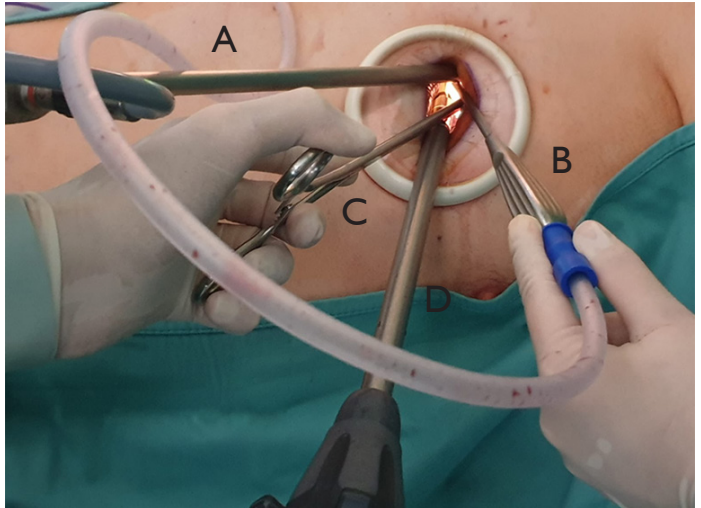

Figure 8 Position of all instruments during operation: (A) camera; (B) suction instrument; (C) grasper and (D) stapler.
A

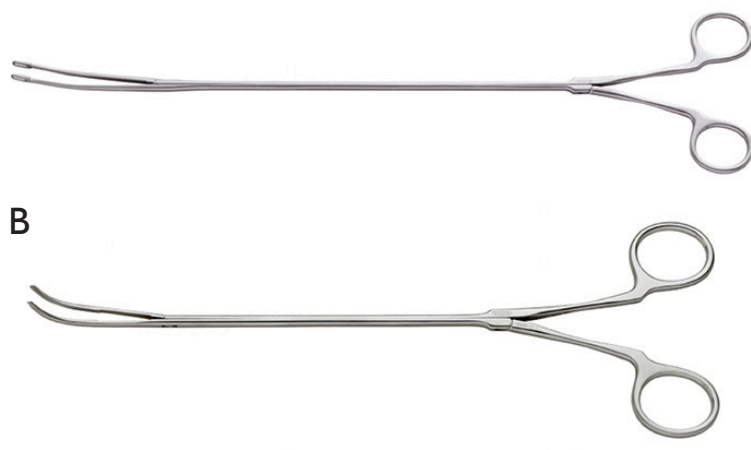

Figure 9 Uniportal VATS most used dissectors. (A) Uniportal VATS "node grasper" used like a blunt tip dissector; (B) uniportal VATS dissector. VATS, video-assisted thoracic surgery.

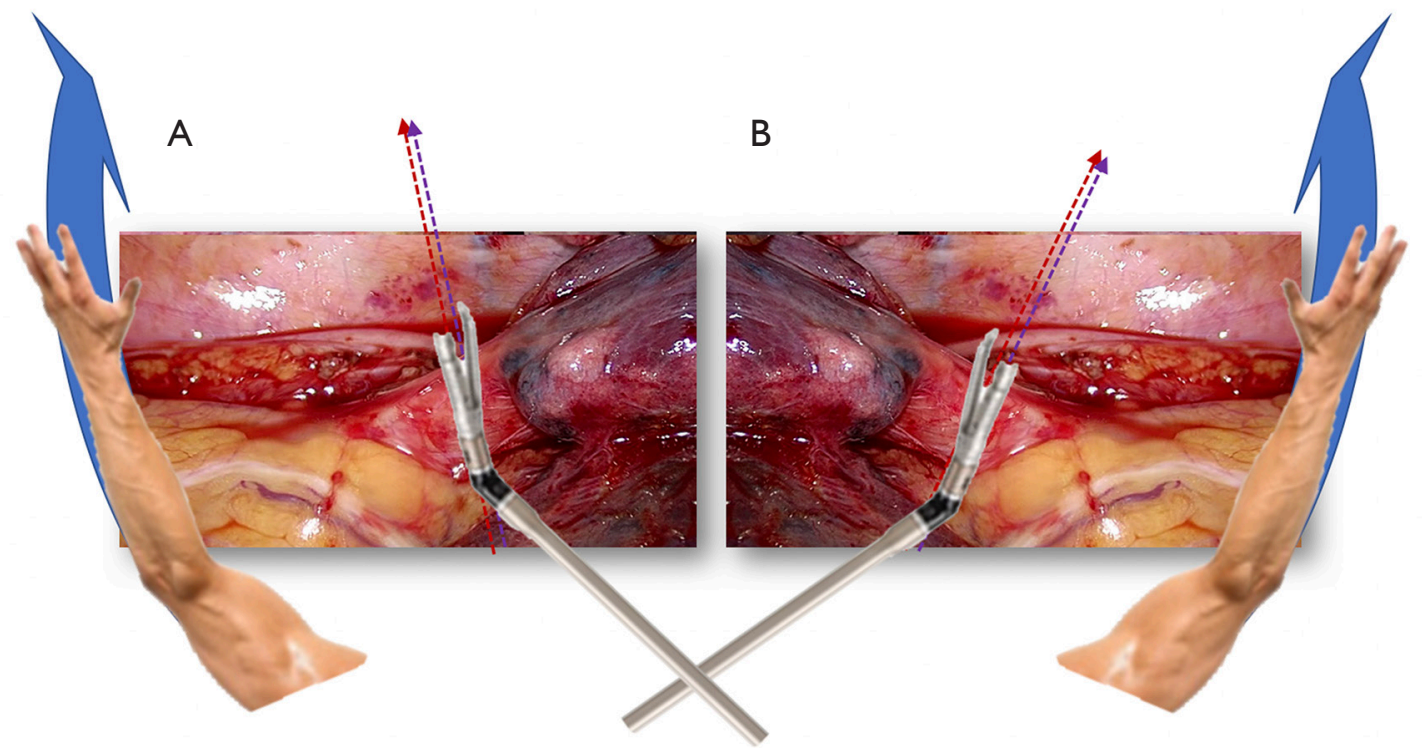

Figure 10 Directions of the tip of the stapler for dissecting the right lower vein (A) and the left lower vein (B).

be caught on segment 7 by clamping and pulling it cranially and towards the back of the patient (14).

The dissection of visceral pleura above the hilum can be performed by using a swab mounted on a clamp or using a uniportal "node grasper" (Figure 9A) like a blunt tip dissector or an energy device for fat pulmonary hila or in case of large hilar lymph-nodes (in the last case a "Maryland jaw" open can be suggested for beginners).

Then the dissection of each hilar structure can be performed by a specific dissector for uniportal VATS (Figure 9B). The section of the hilar structures is done using mechanical or powered endostapler with appropriate vascular or bronchial reloads. In some cases a curved tip reload may help beginners in catching the right angle for difficult veins or arteries.

The stapler-as any dissector or other main working instrument in that moment-must be introduced in the lower part of the incision, with a movement that goes from up to down. Its tip must be curved on the stapler stem in a way to reproduce the direction of the "hand embracing something". For example, for the right side, the operator should think to "embrace the upper hilar structures" opposite him with the right hand and the lower structures with the left hand (Figure 10A), for the left side it is the 

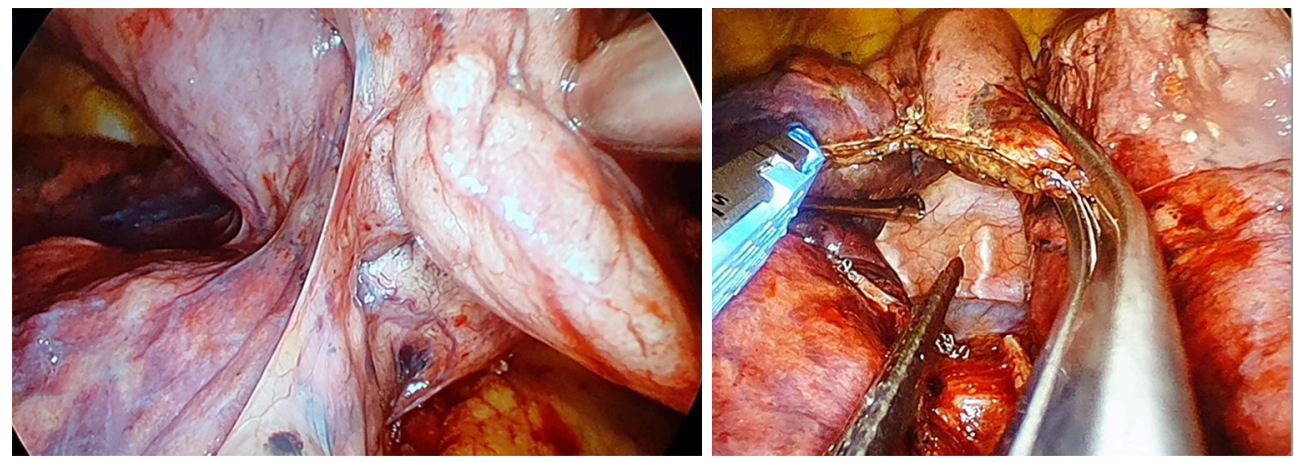

Figure 11 Traction of the upper right lobe for dissecting the fissure in fissure-last technique.

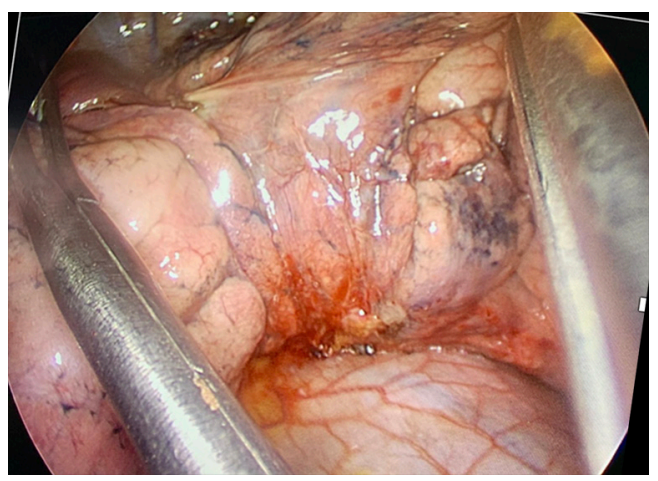

Figure 12 Traction of the lobes on left side in order to expose the artery.

opposite (Figure 10B). The stapler tip must reproduce this movement.

Usually, as in any other VATS technique, it is preferable to close the venous system at the end in order to avoid the blood stasis in the lung. This is usually respected also in uniportal VATS but, sometimes, in case of upper left lobectomy or fissure-less inferior lobectomy, cutting the vena as first step may be helpful for having a better exposure of the arteria or of the bronchus-artery complex, respectively.

In case of fissure-less fissure-last lobectomies, after cutting all hilar structures, the parenchyma of the lobe to be removed must be caught by a grasper and held in a perpendicular way on the horizontal plane, in a position that reproduces quite a "shark fin". In this way, it is quite easy to complete the fissure by endostapler (Figure 11). Attention must be paid to keep all the stumps on the side to be removed.

For lungs with incomplete fissure, the arterial trunk can be easily exposed and prepared by "opening" the upper and lower lobes like if they were "the pages of a book". It can be easily done by using two graspers, one coming from the cranial side of the patient and pushing the lower lobe caudally and backwards, the second coming from the caudal side of the patient and pushing the upper lobe cranially and backwards (Figure 12). In this way, the two graspers work crossed through the incision and are held in the middle part of it, in order to have space for introducing a dissector in the lower part of the incision for preparing the artery.

When the lobectomy is completed, it is suggested to remove the specimen by an endobag. This facilitates the removal of the piece through the small incision and prevents the soiling of neoplastic cells.

For performing lymphadenectomy, it is suggested the use of an energy devices in order to reduce postoperative lymphorrhoea. For performing lymphadenectomy of stations $2-4,5-6$ it is necessary to retract the lung caudally and towards the back of the patient. For station 7 lymphadenectomy, it is useful to open all the posterior pleura, after dividing the ligament, by retracting the lung cranially and towards the anterior mediastinum. Through this explosion, the station 7 is well exposed. Sometimes, pushing the ipsilateral main bronchus down with a swab or using the suction instrument may help in increasing the view of subcarinal area.

\section{Conclusions}

Uniportal VATS is already proven to be a safe and feasible technique, even for complex procedures (17). The use of special dedicated instruments is recommended. Indeed, the standardization of operative room set-up and instrumentation helps to prevent difficulties at the 
beginning and to optimize the results at an advanced stage.

Visiting experienced uniportal VATS centers and attending wet-labs and hands-on courses can reduce the learning curve of this procedure.

\section{Acknowledgments}

None.

\section{Footnote}

Conflicts of Interest: The authors have no conflicts of interest to declare.

Ethical Statement: The authors are accountable for all aspects of the work in ensuring that questions related to the accuracy or integrity of any part of the work are appropriately investigated and resolved.

\section{References}

1. Moisiuc FV, Colt HG. Thoracoscopy: origins revisited. Respiration 2007;74:344-55.

2. Landreneau RJ, Mack MJ, Hazelrigg SR, et al. Videoassisted thoracic surgery: basic technical concepts and intercostal approach strategies. Ann Thorac Surg 1992;54:800-7.

3. Rocco G. One-port (uniportal) video-assisted thoracic surgical resections - a clear advance. J Thorac Cardiovasc Surg 2012;144:S27-31.

4. Roviaro G, Varoli F, Vergani C, et al. Video-assisted thoracoscopic surgery (VATS) major pulmonary resections: the Italian experience. Semin Thorac Cardiovasc Surg 1998;10:313-20.

5. De Leyn P, Dooms C, Kuzdzal J, et al. Revised ESTS guidelines for preoperative mediastinal lymph node staging for non-small-cell lung cancer. Eur J Cardiothorac Surg 2014;45:787-98.

6. Nosotti M, Droghetti A, Luzzi L, et al. First Italian Consensus Conference on VATS lobectomy for NSCLC.

Cite this article as: Ismail $M$, Nachira D. Devising the guidelines: the concept of uniportal video-assisted thoracic surgery-instrumentation and operatory room staff. J Thorac Dis 2019;11(Suppl 16):S2079-S2085. doi: 10.21037/jtd.2019.08.69
Tumori 2017;103:124-35.

7. Howington JA, Blum MG, Chang AC, et al. Treatment of stage I and II non-small cell lung cancer: diagnosis and management of lung cancer, 3rd ed: American College of Chest Physicians evidence-based clinical practice guidelines. Chest 2013;143:e278S-e313S.

8. Rocco G, Martin-Ucar A, Passera E. Uniportal VATS wedge pulmonary resections. Ann Thorac Surg 2004;77:726-8.

9. Yamamoto H, Okada M, Takada M, et al. Video-assisted thoracic surgery through a single skin incision. Arch Surg 1998;133:145-7.

10. Gonzalez D, Paradela M, Garcia J, et al. Single-port videoassisted thoracoscopic lobectomy. Interact Cardiovasc Thorac Surg 2011;12:514-5.

11. Ismail M, Swierzy $M$, Nachira D, et al. Fast-tracking patients through the diagnostic and therapeutic pathways of intrathoracic conditions: the role of uniportal vats. Thorac Surg Clin 2017;27:425-30.

12. Semenkovich TR, Hudson JL, Subramanian M, et al. Enhanced Recovery After Surgery (ERAS) in Thoracic Surgery. Semin Thorac Cardiovasc Surg 2018;30:342-9.

13. Gonzalez-Rivas D. Recent advances in uniportal videoassisted thoracoscopic surgery. Chin J Cancer Res 2015;27:90-3.

14. Ismail $M$, Swierzy $M$, Nachira $D$, et al. Uniportal videoassisted thoracic surgery for major lung resections: pitfalls, tips and tricks. J Thorac Dis 2017;9:885-97.

15. Shariat A, Bhatt H. Successful Use of Serratus Plane Block as Primary Anesthetic for Video-Assisted Thoracoscopic Surgery (VATS)-Assisted Pleural Effusion Drainage. J Cardiothorac Vasc Anesth 2018;32:e31-2.

16. Bertolaccini L, Rocco G, Pardolesi A, et al. The Geometric and Ergonomic Appeal of Uniportal Video-Assisted Thoracic Surgery. Thorac Surg Clin 2017;27:331-8.

17. Wang L, Liu D, Lu J, et al. The feasibility and advantage of uniportal video-assisted thoracoscopic surgery (VATS) in pulmonary lobectomy. BMC Cancer 2017;17:75. 